\title{
In Vitro Interaction Between Oral Hypoglycemic Drug And Herbal Sex Stimulants: Drug Interactions
}

\author{
Md. Nazmul Hossain
}

Department of Biochemistry and Molecular Biology, Faculty of Biological

Sciences,University of Chittagong, Chittagong, Bangladesh

Nishat Akther

Department of Biochemistry and Molecular Biology, Mawlana Bhashani

Science and Technology University, Santosh, Tangail, Bangladesh

\author{
Dr. Md. Alauddin, PhD \\ Robiul Hasan Bhuiyan \\ Muhammad Mosaraf Hossain
}

Department of Biochemistry and Molecular Biology, Faculty of Biological

Sciences, University of Chittagong, Chittagong, Bangladesh

\section{Md. Abdur Rahaman}

Department of Chemistry, Mawlana Bhashani Science and Technology

University, Santosh, Tangail, Bangladesh

\begin{abstract}
Sexual dysfunction is a common, underappreciated complication of diabetes. Male sexual dysfunction among diabetic patients can include disorders of libido, ejaculatory problems, and erectile dysfunction (ED). All three forms of male dysfunction can cause significant bother for diabetic patients and can affect their quality of life. Diabetic patients take oral hypoglycemic drug to control their diabetic as well as take herbal sex stimulants to control to increase the libido. The combined use of herbs and drugs has increased the possibility of herb-drug interactions. The study was undertaken to explore the herb-drug interactions. To investigate the herbdrug interactions an in vitro dissolution study in different simulated $\mathrm{pH}$ medium were performed. In this study gliclazide containing tablet of $80 \mathrm{mg}$ as oral hypoglycemic drug and different herbal sex stimulants available in local market were used. The release mechanism was explored and explained with zero order, first order and Higuchi equations to identify drug interaction. Higher percentage release of gliclazide was found at simulated phosphate buffer of $\mathrm{pH} 7.4$ compared to gastric medium of $\mathrm{pH} 1.2$ and also in presence of herbal sex stimulants. Increased release pattern of gliclazide
\end{abstract}


by concomitant administration of herbal sex stimulants gives slightly higher absorption, which gives the indication of herb-drug interactions.

Keywords: Gliclazide; Herbal sex stimulants; Release kinetics, herb-drug interactions.

\section{Introduction}

Millions of people today use herbal therapies along with prescription and nonprescription medications. Although considered natural, many of these herbal therapies can interact with other medications, causing either potentially dangerous side effects and / or reduced benefits from the medications. Currently, there is very little information published on herbdrug interactions whilst the use of herbs is progressively growing across the world. (Mohammad Yaheya and Ismail; 2009 )A drug interaction occurs when a patient's response to a drug is modified by food, nutritional supplements, formulation excipients, environmental factors, other drugs or disease. Drug interactions cause medications to be less or more potent than intended. Drug interactions can also result in unexpected side effects, which may be harmful. These may occur out of accidental misuse or due to lack of knowledge about the active ingredients involved in the relevant substances (Bushra et al, 2011). Typically, interaction between drugs comes to mind (drug-drug interaction). However, interactions may also exist between drugs $\&$ foods (drug-food interactions), as well as drugs \& herbs (drug-herb interactions). The mechanisms for drug interaction can be divided into several general categories: pharmacokinetics (absorption, distribution, metabolism, and excretion of a drug) and pharmacodynamic interactions (Barbara, 2006). Pharmacokinetic interactions result from alterations in a drug's absorption, distribution, metabolism, or excretion characteristics. These interactions affect drug action by quantitative alterations, either increasing or decreasing the amount of drug available to have an effect. Pharmacodynamic interactions are a result of the influence of combined treatment at a site of biological activity and yield altered pharmacological actions at standard plasma concentrations. Although drug interactions occur through a variety of mechanisms, the effects are the same: the potentiation or antagonism of the effects of drugs. It is difficult to assess the true incidence and clinical significance of drug interactions. Understanding the mechanisms underlying drug interactions is important for the prediction and avoidance of drug toxicity when initiating combination therapy (Stephen, Keith, and Manjunath; 2011).

Diabetes mellitus is a major health problem and an important cause of prolonged ill health and early death (Mutalik and Udupa, 2006). It is a chronic metabolic disorder characterized by hyperglycemia (elevated blood 
glucose level) resulting from defects in insulin secretion, insulin action, or both. It is also characterized by disturbances in carbohydrate, fat and protein metabolism. The vast majority of cases of diabetes fall into two broad etiopathogenetic categories. In one category, type 1 diabetes, the cause is an absolute deficiency of insulin secretion. In the other, much more prevalent category, type 2 diabetes, the cause is a combination of resistance to insulin action and an inadequate compensatory insulin secretory response.Long-term complications of diabetes include retinopathy with potential loss of vision; nephropathy leading to renal failure; peripheral neuropathy with risk of foot ulcers, amputations, and Charcot joints; and autonomic neuropathy causing gastrointestinal, genitourinary, and cardiovascular symptoms and sexual dysfunction. Patients with diabetes have an increased incidence of atherosclerotic cardiovascular, peripheral arterial, and cerebrovascular disease. Type 2 diabetes is the most common form of diabetes mellitus, accounting for approximately $90 \%$ of cases and affecting about 100 million people in the word. Projection indicates that there will be over 450 million type 2 diabetic patients by the year 2030 (International Diabetes Federation, 2011. Wild et al, 2004). Men with diabetes tend to develop erectile dysfunction 10 to 15 years earlier than men without diabetes. As men with diabetes age, erectile dysfunction becomes even more common. Though diabetes and Erectile Dysfunction are two separate conditions, they tend to go hand-in-hand. According to the National Institute of Diabetes and Digestive and Kidney Diseases, men with diabetes are two to three times more likely to develop erectile dysfunction. In many cases, problems with achieving or maintaining an erection is an early clue that you may be diabetic, particularly in men 45 and under. Diabetes mellitus is a metabolic disorder in which prolonged treatment is necessary. Maintenance of normal blood glucose level is essential in this condition, since both hyperglycemias as well as hypoglycemia are unwanted effects. An ideal antidiabetic drug would be the one that not only does control the glycemia level but also prevents the development of complications (Schernthaner, 2003). Oral hypoglycemic agents like sulphonylureas are still the major players in the management of type 2 diabetes. Gliclazide is an oral hypoglycemic (antidiabetic drug) and is classified as a sulfonylurea. Its classification has been ambiguous, as literature uses it as both a first generation (Ballagi et al., 1990) and second generation (Shimoyama et al., 2006) sulfonylurea. Gliclazide was shown to protect human pancreatic betacells from hyperglycemia induced apoptosis (Del Guerra et al., 2007). It was also shown to have an antiatherogenic effect (preventing accumulation of fat in arteries) in type 2 diabetes. (Katakami et al., 2004). Gliclazide is used when diabetes cannot be controlled by proper dietary management and exercise or when insulin therapy is not appropriate. Gliclazide is available as oral tablets 
(30 and $80 \mathrm{mg}$ strength) with the recommended dosage between 40 and $320 \mathrm{mg} /$ day. Reports from the in vivo studies show that, after oral administration, gliclazide is almost completely absorbed (Delrat et al, 2002; Najib et al, 2002). However, due to its low and $\mathrm{pH}$-dependent aqueous solubility (Hong et al, 1998; Özkan et al, 2000; Shewale et al, 2008), Gliclazide absorption rate appears to be slow and variable (Hong et al, 1998; Kobayashi et al, 1981; Davis et al, 2000; Rana, 2010), and therefore, its absorption profile is difficult to decipher.

Herb is plant or plant part used for its scent, flavor, or therapeutic properties. Herbal medicines are one type of dietary supplement. They are sold as tablets, capsules, powders, teas, extracts, and fresh or dried plants. People use herbal medicines to try to maintain or improve their health. Many people believe that products labeled "natural" are always safe and good for them. This is not necessarily true. Herbal medicines do not have to go through the testing that drugs do. Some herbs, such as comfrey and ephedra, can cause serious harm. Some herbs can interact with prescription or overthe-counter medicines. Herbal sex stimulants for Men contains a selection of herbs (John, 2007) from around the world and which are traditionally identified as having aphrodisiac properties and are safe and non-addictive. They are known for their supportive function in maintaining sexual health and well-being. Herbal sex stimulants for men is used for strong erections without risk of side effects, maintain sexual arousal, drive and desire, promotes systemic and hormonal balance. Male impotence also called Erectile dysfunction is considered as one of the most important public health problems, since it affects a great percentage of men worldwide. Erectile dysfunction is defined as the consistent inability to obtain or maintain an erection for satisfactory sexual relations. An estimated 20-30 million men suffer from some degree of sexual (Gallé and Trummer, 2003). It occurs commonly in middle-aged and older men. Impotence occurs in $50 \%$ of men with diabetes mellitus. Erectile dysfunction is also associated with some therapeutic agents like antihypertensives, antipsychotics, antidepressants, and drugs for diabetes mellitus (Pallavi et al., 2011; Guay et al., 20003; Martey, 2010).Treatment of Erectile dysfunction involves several natural aphrodisiac potentials. Aphrodisiac is described as any substance that enhances sexual pleasure.Plants like Bhindi, Musk mallow, Peanut, Garlic, Chiense chive, Punarnava, Capsicum, Coconut, Carrot, Ginseng, Ginger, West African Pepper, Gingembre and many other herbs found worldwide has aphrodisiac properties, hence used as sex stimulants for men (Pallavi et al., 2011). Herbal sex stimulants are found in different dosage forms like tablets, capsules, powder, and cream.

Therefore, the objective of the present work is to identify any drug interaction between oral hypoglycemic drugs with herbal sex stimulants. 
Drug-herbal interactions can occur at the pharmaceutical, pharmacodynamic or pharmacokinetic levels (Beijnen and Schellens, 2004) but most of the interactions occur at pharmacokinetic level (Brazier and Levine, 2003) that involves changes in absorption, distribution, metabolism and excretion of the conventional drug, which in turn determine the bioavailability of the drug. The use of adulterated herbal medicines sometimes result in serious ailments such as acute renal failure (De Smet, 1995; Abt et al., 1995; Nelson et al., 1995; Gertner et al., 1995; Van et al., 1994). In the present study, release kinetics of oral hypoglycemic drug (e.g. glyclazide) was determined alone and in presence herbal sex stimulants to understand the drug interactions.

\section{Materials, methods and equipments Materials}

Gliclazide (designated as Gli) tablets of one brand and the innovator brand with labeled contents of $80 \mathrm{mg}$ and five different herbal sex stimulants tablets (designated as $\mathrm{H} 1, \mathrm{H} 2, \mathrm{H} 3, \mathrm{H} 4, \mathrm{H} 5$, and $\mathrm{H} 6$ ) for men according to Bangladesh National Formulary of Unani Medicine were obtained from local market of Bangladesh. All the drugs were checked for their production and expiry dates. Purified water used throughout the research. Other materials used for analysis were also purchased from local market and these were all analytical grade.

\section{List of Equipments}

- $\quad$ Electronic balance

- $\quad$ USP Dissolution apparatus - II

- UV Visible spectrometer

\section{In vitro Dissolution study}

In-vitro dissolution study of gliclazide and gliclazide with herbal drugs was carried out in a USP Type II (paddle type) dissolution apparatus. Simulated gastric medium of $\mathrm{pH} 1.2$ and Phosphate buffer $\mathrm{pH} 7.4$ were used in separate experiment as dissolution medium that was maintained at $37 \pm$ $0.5^{\circ} \mathrm{C}$ and stirred at 100 revolutions per minutes (rpm). At predetermined time intervals, 10-ml sample was withdrawn and replaced with fresh dissolution medium. After filtration and appropriate dilution, the sample solution was analyzed at $226 \mathrm{~nm}$ and $290 \mathrm{~nm}$ using respective blank solution in a reference cell. Correct the absorbance obtained at $226 \mathrm{~nm}$ by subtracting the absorbance obtained at $290 \mathrm{~nm}$. The dissolution study was continued for 8 hours in each experiment to get a simulated picture of the drug release in the in-vivo condition. The drug release profile was fitted into several mathematical models to get an idea of the release mechanism and drug interaction. 


\section{Kinetics modeling of drug release}

Different kinetic models (zero order, first order and Higuchi equation) were applied to analyze the mechanism of drug release-rate kinetics. Mathematical modeling increases understanding of the release mechanism and in turn helps to identify drug interactions. Here, in vitro drug release data obtained from the dissolution studies of gliclazide tablets only and gliclazide with herbal sex stimulants were fitted to zero order, first order and Higuchi equation to ascertain the kinetic modeling of drug release and drug interaction.

\section{Zero Order Kinetics}

Zero-order (Wagner, 1969) release kinetics describes systems where the drug release rate is constant over a period of time and it is the ideal method of drug release in order to achieve a pharmacological prolonged action. The zero-order release kinetics can, in a simple way, express by following equation:

Where,

$$
Q_{t}=Q_{o}+K_{o} t
$$

$$
\begin{aligned}
& \mathrm{Q}_{\mathrm{t}}=\text { amount of drug released in time ' } \mathrm{t} \text { ' } \\
& \mathrm{Q}_{\mathrm{o}}=\text { initial amount of drug in the solution, } \\
& \mathrm{K}_{\mathrm{o}}=\text { zero order release constant }
\end{aligned}
$$

A plot of amount of drug released versus time will be linear for zeroorder kinetics. The dosage forms following this profile, release the same amount of drug by unit time and it is the ideal method of drug release in order to achieve a prolonged pharmacological action. This relation can be used to determine the drug dissolution from various types of modified release dosage forms such as matrix tablets with low soluble drugs, coated tablets and capsules and osmotic systems.

\section{First Order Kinetics}

The application of this model to drug dissolution studies was first proposed by Gibaldi and Feldman (1967) and later by Wagner (1969). In typical first-order release kinetics, the drug release rate depends on its concentration. The first order release kinetics can be expressed by following equation:

Where,

$$
\log \mathrm{Q}_{\mathrm{t}}=\log \mathrm{Q}_{\mathrm{o}}+\mathrm{K}_{\mathrm{t}} / 2.303
$$

$\mathrm{Q}_{\mathrm{t}}=$ amount of drug released in time ' $\mathrm{t}$ '.

$\mathrm{Q}_{0}=$ initial amount of drug in the solution,

$\mathrm{K}_{\mathrm{t}}=$ first order release constant

Here, the graphical representation of the log cumulative of \% drug remaining versus time will be linear with a negative slope. The dosage form 
follows this profile such as those containing water soluble drug in a porous matrices, release the drug that is proportional to the amount of drug released by unit time diminish.

\section{Higuchi Model}

A descriptive mathematical model for drug dissolution from matrix systems was not developed until 1961 by Higuchi. The model was initially derived for planar systems; however it has since been modified for use with different geometries and porous systems. The most familiar form of the Higuchi model is the simplified Higuchi model, which relates drug concentration to the square root of time:

Where,

$$
f_{t}=K_{H} t^{1 / 2}
$$

$\mathrm{f}_{\mathrm{t}}=$ Concentration of drug in the drug matrix at time ' $\mathrm{t}$ '

$\mathrm{K}_{\mathrm{H}}=$ Higuchi dissolution constant

\section{Results and discussion}

To investigate the drug interaction of oral hypoglycemic drug (e.g. gliclazide) with herbal sex stimulants for men we performed in vitro dissolution study and different kinetic model also studied. Oral administration of gliclazide and concomitant administration of herbal sex stimulants might bring some relative changes in release kinetics of gliclazide.

\section{Kinetic modeling of drug release}

Drug release data obtained were extrapolated by Zero order, First order and Higuchi equations to know the mechanism of drug release and drug interaction.

In case of Zero order rate kinetics (cumulative amount of drug released versus time; Figure-1 a, b), it was observed that the percent release of gliclazide increased with time (for 8 hours) at simulated $\mathrm{pH}$ medium of $1.2 \&$ 7.4. A burst release observed within first hour. Higher percent release of gliclazide observed at $\mathrm{pH} 7.4$ than $\mathrm{pH} 1.2$. Slightly higher percent release of gliclazide also observed in presence of herbal sex stimulants than than gliclazide alone.

In case of First order rate kinetics (log cumulative percentage of drug remaining versus time; Figure-1 c, d), it was observed that the log of percent remaining of gliclazide decreased with time (for 8 hours). It is also observed that in presence of herbal sex stimulants log of percent remaining of gliclazide decreased more with time at different simulated $\mathrm{pH}$ medium of $\mathrm{pH}$ 1.2 and $\mathrm{pH} 7.4$. 
In case of Higuchi release kinetics (cumulative percentage of drug released versus square root of time; Figure-e, f) it was observed that the percent release of gliclazide and gliclazide in presence of herbal sex stimulants increased with square root of time (for 8 hours) at different simulated $\mathrm{pH}$ medium ( $\mathrm{pH} 1.2$ and $\mathrm{pH}$ 7.4). Slightly higher percent release of gliclazide observed in presence of herbal sex stimulants than that of gliclazide alone.

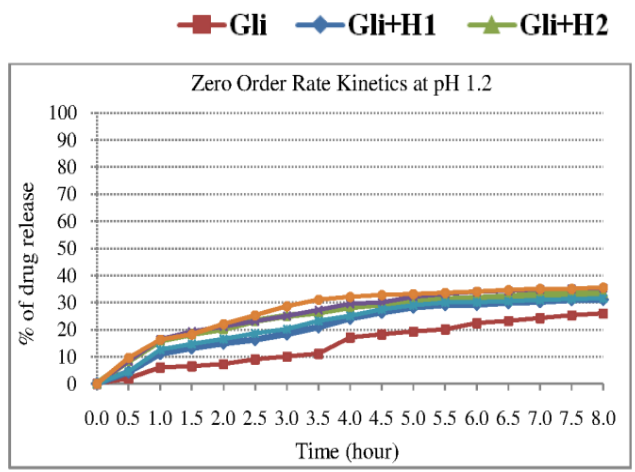

(a)

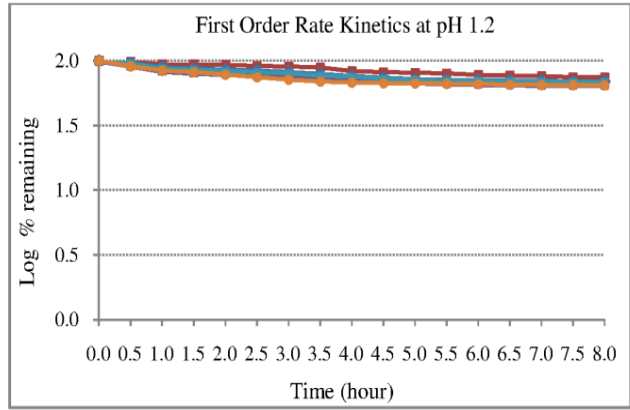

(c)

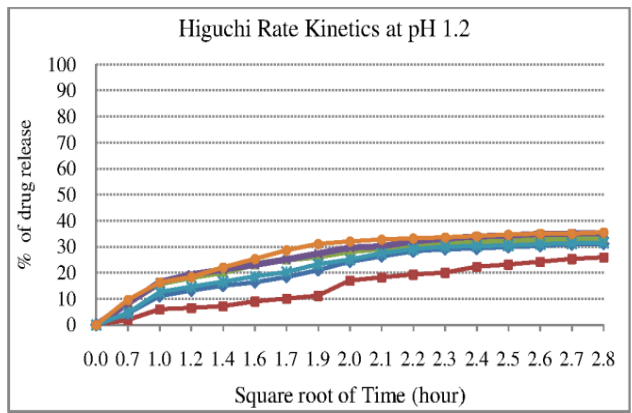

(e)

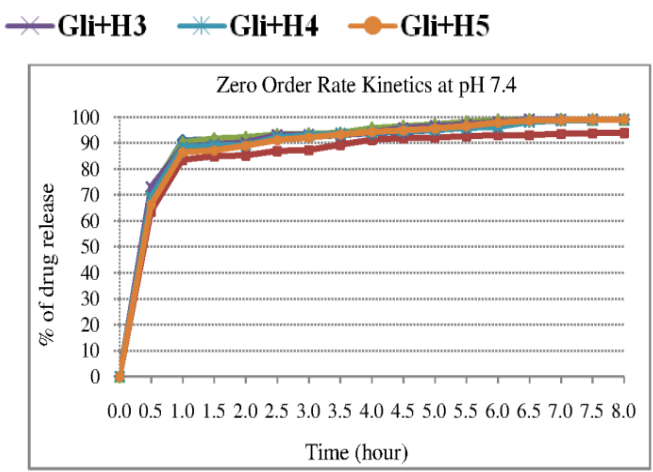

(b)

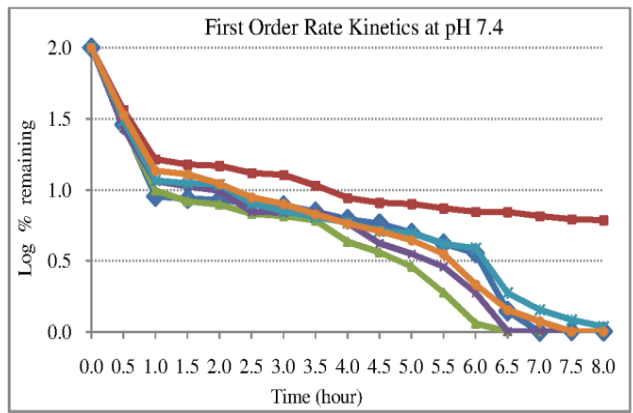

(d)

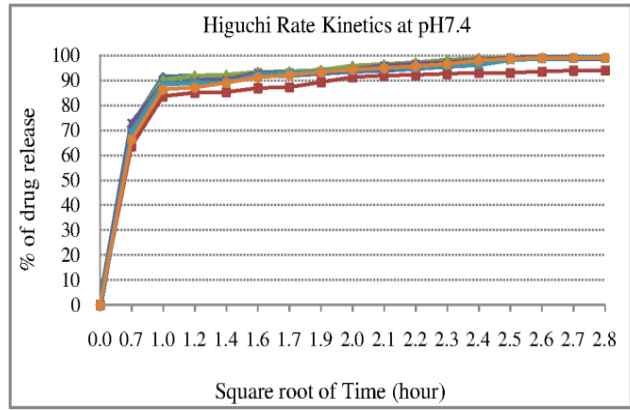

(f)

Figure-1: Zero order release model $(a, b)$; First order release model $(c, d)$; Higuchi release model $(e, f)$ of gliclazide and gliclazide in presence of herbal sex stimulants at simulated medium of pH1.2 \& pH 7.4. Coffee color line indicating the release pattern of Gliclazide only and others five lines of different color indicating the release pattern of Gliclazide in the presence of five different herbal sex stimulants. 


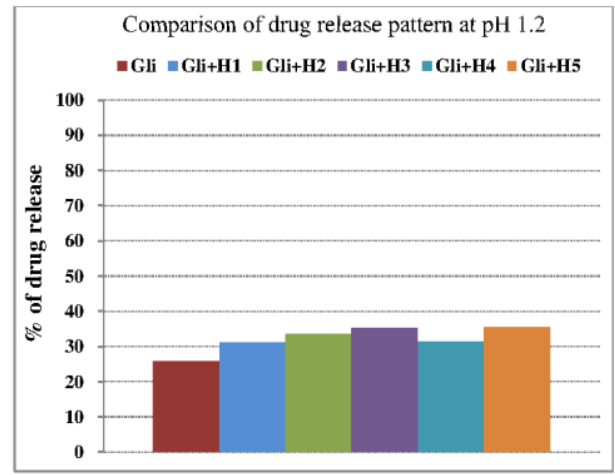

(a)

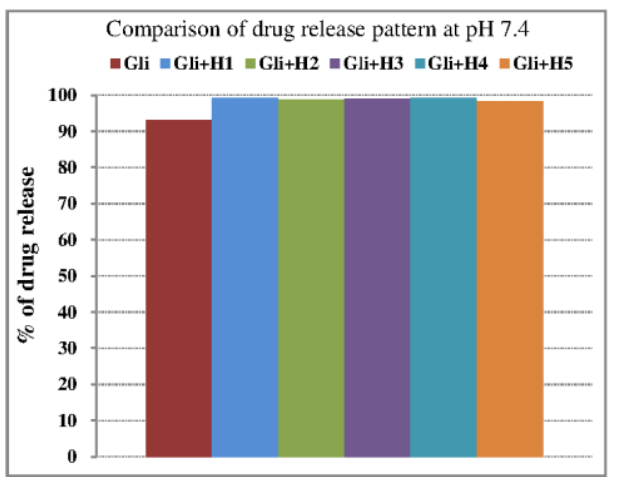

(b)

Figure-2: Bar diagram representing the comparison of release pattern of gliclazide (coffee color bar) and gliclazide in presence of herbal sex stimulants (other five bars of different colors) at simulated medium of $\mathrm{pH}$ 1.2 (a) and $\mathrm{pH} 7.4$ (b) for a period of 8 hours.

From the results (Figure-1 \& 2) it is clearly demonstrated that the percent $(\%)$ release gliclazide increased with time and a burst release observed within first hour. It is also observed that the percent release of gliclazide increased more in presence of herbal sex stimulants as well as with the increase of $\mathrm{pH}$ which indicated that the increase in absorption of gliclazide in presence of herbal sex stimulants. But slightly higher percent release of gliclaide observed in presence of different herbal sex stimulants at the present study. Since the percent $(\%)$ release of gliclazide was not significantly changed in presence of herbal sex stimulants comparing to gliclazide alone, which gives us an idea that if we takes this two drug concurrently there will be no hazardous effect from each other.

\section{Conclusion \& future plan}

According to the release study, the higher percent release of oral hypoglycemic drug (gliclazide) in presence of different herbal sex stimulants at different simulated medium $(\mathrm{pH} 1.2$ and $\mathrm{pH}$ 7.4) indicating a drug interaction. But the significant difference of percent release between oral hypoglycemic drug (gliclazide) and oral hypoglycemic drug (gliclazide) in presence of herbal sex stimulants is not observed; suggesting the interaction is not harmful. It is difficult to assess the true incidence and clinical significance of drug interactions. Understanding the mechanisms underlying drug interactions is important for the prediction and avoidance of drug toxicity when initiating combination therapy. The process of using in vitro models to predict in vivo drug interactions is still in its infancy, and extensive validation of this approach is needed. In vitro models predictive of in vivo drug interactions will be essential for rapid, cost-effective screening of pharmaceutical compounds and are important for reducing risks to patient safety. So further wide scale study is necessary underlying the present drug interaction. 


\section{References:}

Abt AB, Oh JY, Huntington RA, Burkhart KK. Chinese herbal medicine induced acute renal failure. Arch Intern Med. 1995 Jan 23; 155(2):211-2.

Ballagi-Pordány, György; Köszeghy, Anna; Koltai, MáriaZsófia; Aranyi, Zoltán; Pogátsa, Gábor (1990). "Divergent cardiac effects of the first and second generation hypoglycemic sulfonylurea compounds". DiabetesResearch and Clinical Practice 8 (2): 109-14. doi:10.1016/01688227 (90) 90020T. PMID 2106423.

Bangladesh National Formulary of Unani Medicine.

Barbara J Pleuvry, 2006, Pharmacodynamic and pharmacokinetic drug interactions, 13 November 2006.

Beijnen JH, Schellens JH (2004). Drug interactions in oncology. Lancet Oncol 5: 489-496.

Brazier NC, Levine MA (2003). Drug-herb interaction among commonly used conventional medicines: a compendium for health care professionals. Am J Ther 10: 163-169.

Bushra R, Aslam N, Khan AY. Food-drug interactions. Oman Med J. 2011 Mar; 26(2):77-83

Davis TME, Daly F, Walsh JP, Ilett KF, Beilby JP, Dusci LJ, et al. Pharmacokinetics and pharmacodynamics of gliclazide in Caucasians and Australian Aborigines with type 2 diabetes. $\mathrm{Br} \mathrm{J}$ Clin Pharmacol. 2000;49:223-230. doi: 10.1046/j.1365-2125.2000.00162.x.

[PMC free article] [PubMed][Cross Ref]

De Smet PAGM. Health risks of herbal remedies. Drug Safety. 1995; 13:8193.

Del Guerra, S; Grupillo, M; Masini, M; Lupi, R; Bugliani, M; Torri, S; Boggi, U; Del Chiaro, M; et al. (2007). "Gliclazide protects human islet betacells from apoptosis induced by intermittent high glucose". Diabetes/Metabolism Research and Reviews 23 (3): 234-8. doi:10.1002/dmrr.680. PMID 16952202.

Delrat P, Paraire M, Jochemsen R. Complete bioavailability and lack of food-effect on pharmacokinetics of gliclazide $30 \mathrm{mg}$ modified release in healthy volunteers. Biopharm Drug Dispos. 2002;23:151-157. doi: 10.1002/bdd.303. [PubMed] [Cross Ref]

Gallé G, Trummer H. The etiology of erectile dysfunction and mechanisms by which drugs improve erection. Drugs Today (Barc) 2003;39:193202. [PubMed]

Gertner, E., Marshall, P.S., Filandrinos, D., Potek, A.S., Smith, T.M. Complications resulting from the use of Chinese herbal medications containing undeclared prescription drugs. Arthritis Rheum 1995; 38; 614-7. Gibaldi, M.; Feldman, S. Establishment of sink conditions in dissolution rate determinations-theoretical considerations and application to non- 
disintegrating dosage forms. J. Pharm. Sci., Washington, v.56, p.1238- 1242, 1967.

Guay AT, Spark RF, Bansal S, Cunningham GR, Goodman NF, Nankin HR, et al. American Association of Clinical Endocrinologists medical guidelines for clinical practice for the evaluation and treatment of male sexual dysfunction: A couple's problem - 2003 update. Endocr Pract. 2003;9:7795.[PubMed]

Higuchi, T. Mechanism of sustained release medication: theoretical analysis of rate of release of solid drugs dispersed in solid matrices. J. Pharm. Sci., Washington, v.52, p.1145-1149, 1963.

Hong SS, Lee SH, Lee YJ, Chung SJ, Lee MH, Shim CK. Accelerated oral absorption of gliclazide in human subjects from a soft gelatin capsule containing a PEG 400 suspension of gliclazide. J Control Release.1998;51:185-192.doi:10.1016/S0168-3659(97)001673. [PubMed] [Cross Ref]

International Diabetes Federation. [Updated 2011 Mar 28; cited 2011 Jun 15]. Available from:URL:http://www.idf.org/media-events/press-release/idfannounces-new-position-supporting-surgery-treat-type-2-diabetes.

John Year Ital, 2007, Powerful Sex Foods and Stimulants, Page 1 to 26.

Katakami, N.; Yamasaki, Y.; HayaishiOkano, R.; Ohtoshi, K.; Kaneto, H.; Matsuhisa, M.; Kosugi, K.; Hori, M. (2004). "Metformin or gliclazide, rather than glibenclamide, attenuate progression of carotid intimamedia thickness in subjects with type 2 diabetes". Diabetologia 47 (11): 1906-13. doi:10.1007/s0012500415478. PMID 15565373.

Kobayashi K, Kimura M, Sakoguchi T, Kitani Y, Hata M, Matsuoka A. Influence of blood proteins on biomedical analysis. III. Pharmacokinetics and protein binding of gliclazide. J Pharm Dyn. 1981;4:436-442.[PubMed] Martey ON, He X. Possible mode of action of Mondia whitei: An aphrodisiac used in the management of erectile dysfunction. J Pharmacol Toxicol. 2010;5:460-8.

Mohammad Yaheya, Mohammad Ismail. Herb-drug interactions and patient counseling- Review Article. International Journal of Pharmacy and Pharmaceutical Sciences, Vol. 1, Supply 1, Nov.-Dec. 2009.

Mutalik S, Udupa N. Pharmacological evaluation of membranemoderated transdermal system of glipizide. Clin. Exp. Pharmacol. Physiol. . 2006;33:17-26. [PubMed]

Najib N, Idkaidek N, Beshtawi M, Bader M, Admour I, Mahmood Alam S, et al. Bioequivalence evaluation of two brands of gliclazide $80 \mathrm{mg}$ tablets (Glyzide $^{\circledR}$ \& Diamicron ${ }^{\circledR}$ )-in healthy human volunteers. Biopharm Drug Dispos. 2002;23:197-202. doi: 10.1002/bdd.310. [PubMed] [Cross Ref]

Nelson L, Shih R, Hoffman R.; Aplastic anemia induced by an adulterated herbal medication. J Toxicol Clin Toxicol. 1995; 33(5):467-70. 
Özkan Y, Atay T, Dikmen N, Işimer A, Aboul-Enein HY. Improvement of water solubility and in vitro dissolution rate of gliclazide by complexation with $\beta$-cyclodextrin. Pharm Acta Helv. 2000;74:365-370. doi: 10.1016/S0031-6865(99)00063-1. [PubMed] [Cross Ref]

Pallavi K.J, Ramandeep Singh , Sarabjeet Singh, Karam Singh, Mamta Farswan, Vinod Singh. Aphrodisiac agents from Medicinal Plants: A Review. J. Chem. Pharm. Res., 2011, 3(2):911-921

Rana MKZ. Gliclazide and glibenclamide interactions with antacids and $\mathrm{H}_{2}-$ antagonists. Ph.D Thesis, Department of Chemistry, University of Karachi, Pakistan.2003.http://prr.hec.gov.pk/Chapters/973-1.pdf. Accessed 23 Jun 2010.

Schernthaner G. Gliclazide modified release: A critical review of pharmacodynamic, metabolic andvasoprotective effects. Metabolism. 2003;52:29-34. [PubMed]

Shimoyama, Tatsuhiro; Yamaguchi, Shinya; Takahashi, Kazuto; Katsuta, Hidenori; Ito, Eisuke; Seki, Hiroyuki; Ushikawa, Kenji; Katahira, Hiroshi; et al. (2006). "Gliclazide protects 3T3L1 adipocytes against insulin resistance induced by hydrogen peroxide with restoration of GLUT4 translocation". Metabolism 55 (6): 722-30. doi:10.1016/j.metabol.2006.01.019. PMID 16713429.

Shewale BD, Fursule RA, Sapkal NP. Effect of pH and hydroxylpropyl- $\beta$ cyclodextrin on solubility and stability of gliclazide. Int $J$ Health Res. 2008;1:95-99.

Stephen C. Piscitelli, Keith A. Rodvold, Manjunath P. Pali. Book Title: Drug Interactions in Infectious Diseases; Springer New York Dordrecht Heidelberg London. Library of Congress. Copyright: 2011; DOI: 10.1007/978-1-61779-213-7.

Van der Stricht, B.I., Parvais, O.E., Vanhaelen-Fastre, R.J., and Vanhaelen, M.H., Remedies may contain cocktail of active drugs. Br. Med. J., 308, 1162, 1994.

Wagner, J.G. Interpretation of percent dissolved-time plots derived from in vitro testing of conventional tablets and capsules. J. Pharm. Sci., Washington, v.58, p.1253-1257, 1969.

Wild S, Roglic G, Green A, Sicree R, King H. Global prevalence of diabetes: estimates for the year 2000 and projections for 2030. Diabetes Care. 2004;27:1047-1053. [PubMed] 